\title{
THEORETICAL TOPICS IN SPACETIME-SYMMETRY VIOLATIONS
}

\author{
R. LEHNERT \\ Center for Theoretical Physics \\ Massachusetts Institute of Technology \\ Cambridge, MA 02139, USA \\ E-mail: rlehnert@lns.mit.edu
}

\begin{abstract}
The Lorentz- and CPT-violating Chern-Simons extension of electrodynamics is considered. In the context of $N=4$ supergravity in four spacetime dimensions, it is argued that cosmological solutions can generate this extension. Within ChernSimons electrodynamics, theoretical and phenomenological topics are reviewed that concern the number of the remaining spacetime symmetries and the vacuum Cherenkov effect, respectively.
\end{abstract}

\section{Introduction}

Spacetime-symmetry investigations owe their present popularity to the idea that Lorentz- and CPT-violation could be a signature from unknown physics possibly arising at the Planck scale. Research in this field may therefore be divided into three broad and overlapping areas: the identification of mechanisms that can generate Lorentz and CPT breakdown in underlying physics, the determination and theoretical study of the ensuing low-energy effects, and the low-energy phenomenology together with the corresponding experimental tests.

During the last two decades, a number of theoretical arguments suggesting the possibility of spacetime-symmetry breaking in underlying physics have been put forward. Examples of such arguments involve string field theory, ${ }^{1}$ realistic noncommutative field theories, ${ }^{2}$ spacetime-varying fields, ${ }^{3}$ various quantum-gravity models, ${ }^{4}$ nontrivial spacetime topology, ${ }^{5}$ randomdynamics models, ${ }^{6}$ multiverses,${ }^{7}$ and brane-world scenarios. ${ }^{8}$ Although the underlying dynamics remains Lorentz invariant in most of the above situations, Lorentz and CPT symmetry are nevertheless violated in the ground state at low energies. These ideas provide one of key motivations for Lorentz- and CPT-violation research. 
At presently attainable energies, the effects resulting from Lorentz and CPT breakdown in underlying physics are described by the Standard-Model Extension (SME) - an effective-field-theory framework containing the usual Standard Model $^{9}$ and general relativity. ${ }^{10}$ Various theoretical investigations and consistency analyses have been performed within the context of the SME. ${ }^{11,12}$ While some of these studies have clarified conceptual issues, non have suggested any internal inconsistencies.

The SME has also provided the basis for numerous phenomenological and experimental investigations of Lorentz and CPT violation. ${ }^{13}$ Specific analyses include, for example, ones with photons, ${ }^{14,15}$ neutrinos, ${ }^{16}$ electrons, ${ }^{17}$ protons and neutrons, ${ }^{18}$ mesons, ${ }^{19}$ and muons ${ }^{20}$ These studies have placed tight constraints on numerous SME coefficients or combinations of them. Some of the obtained bounds can be considered to probe the Planck scale.

The above remarks demonstrate that all three of the aforementioned areas of Lorentz- and CPT-violation research are active and vibrant fields of scientific inquiry spanning many physics disciplines. This talk aims at illustrating within a specific example - namely the Maxwell-Chern-Simons (MCS) model $^{21}$ contained in the SME - how the three subfields are interwoven. Section 2 shows that the Lorentz- and CPT-violating MCS model can arise in underlying Lorentz-invariant physics, more specifically in a lowenergy cosmological context of $N=4$ supergravity. In Sec. 3, some theoretical issues regarding the counting of symmetries in the MCS model are discussed at the level of the SME. A review of vacuum Cherenkov radiation, which is a phenomenological effect occuring in MCS theory, is presented in Sec. 4

\section{Emergence of the MCS model in supergravity cosmology}

The discussion in this section is based upon results obtained in the first paper of Ref. 3, which considers pure $N=4$ supergravity in four spacetime dimensions. Although unrealistic in detail, it is a limit of $N=1$ supergravity in eleven dimensions, which is contained in M-theory. We may thus expect that the model can nevertheless illuminate generic aspects of a candidate underlying theory.

When only one of the model's graviphotons, $F^{\mu \nu}$, is excited, the bosonic part of our model is given by

$$
\begin{array}{r}
\kappa \mathcal{L}_{\mathrm{sg}}=-\frac{1}{2} \sqrt{g} R+\sqrt{g}\left(\partial_{\mu} A \partial^{\mu} A+\partial_{\mu} B \partial^{\mu} B\right) / 4 B^{2} \\
-\frac{1}{4} \kappa \sqrt{g} M F_{\mu \nu} F^{\mu \nu}-\frac{1}{4} \kappa \sqrt{g} N F_{\mu \nu} \tilde{F}^{\mu \nu},
\end{array}
$$


where $M$ and $N$ are known functions of the scalars $A$ and $B, g=-\operatorname{det}\left(g_{\mu \nu}\right)$, and $\tilde{F}^{\mu \nu}=\varepsilon^{\mu \nu \rho \sigma} F_{\rho \sigma} / 2$. We can rescale $F^{\mu \nu} \rightarrow F^{\mu \nu} / \sqrt{\kappa}$ removing the explicit appearance of the gravitational coupling $\kappa$ from the equations of motion. We represent the model's fermions by the energy-momentum tensor of dust $T_{\mu \nu}=\rho u_{\mu} u_{\nu}$ modeling, e.g., galaxies. Here, $u^{\mu}$ is a unit timelike vector and $\rho$ is the fermionic energy density. At tree level, the fermionic matter is uncoupled from the scalars, so that $T_{\mu \nu}$ is conserved separately.

With the phenomenological input of an isotropic homogeneous flat Friedmann-Robertson-Walker Universe, we can take $F^{\mu \nu}=0$ on large scales. Our cosmology then obeys the Einstein equations and the equations of motion for the scalars $A$ and $B$. These equations can be solved analytically yielding a nontrivial dependence of $A=A_{\mathrm{b}}(t)$ and $B=B_{\mathrm{b}}(t)$ on the comoving time $t$. Consider now small localized excitations of $F_{\mu \nu}$ in the scalar background $A_{\mathrm{b}}$ and $B_{\mathrm{b}}$. The effective Lagrangian $\mathcal{L}_{\text {cosm }}$ for such situations in local inertial coordinates follows from Eq. (1) and is

$$
\mathcal{L}_{\text {cosm }}=-\frac{1}{4} M_{\mathrm{b}} F_{\mu \nu} F^{\mu \nu}-\frac{1}{4} N_{\mathrm{b}} F_{\mu \nu} \tilde{F}^{\mu \nu},
$$

where $A_{\mathrm{b}}(t)$ and $B_{\mathrm{b}}(t)$ imply the time dependence of $M_{\mathrm{b}}$ and $N_{\mathrm{b}}$. Comparison with the usual Maxwell Lagrangian $\mathcal{L}_{\mathrm{em}}=-\frac{1}{4 e^{2}} F_{\mu \nu} F^{\mu \nu}-\frac{\theta}{16 \pi^{2}} F_{\mu \nu} \tilde{F}^{\mu \nu}$ establishes that $e^{2} \equiv 1 / M_{\mathrm{b}}$ and $\theta \equiv 4 \pi^{2} N_{\mathrm{b}}$. Thus, $e$ and $\theta$ acquire time dependencies via the varying background $A_{\mathrm{b}}$ and $B_{\mathrm{b}}$.

The time dependence of $e$ is an interesting topic in itself, but in the present context the goal is to obtain the Lorentz- and CPT-violating ChernSimons term $\left(k_{A F}\right)^{\mu} A^{\nu} \tilde{F}_{\mu \nu}$ contained in the SME. This can be achieved at the level of the action via an integration by parts of the $\theta$-angle term. This establishes the desired result

$$
\mathcal{L}_{\text {cosm }} \supset \frac{1}{2}\left(\partial_{\mu} N_{\mathrm{b}}\right) A_{\nu} \tilde{F}^{\mu \nu} .
$$

It is thus apparent that, starting from a Lorentz-invariant model, our supergravity cosmology has indeed generated one particular SME operator.

\section{Symmetry counting in the MCS model}

In addition to the usual ten Poincaré invariances (four translations, three rotations, and three boosts), conventional electrodynamics possesses five further spacetime symmetries: one dilatation and four special conformal transformations. The inclusion of our Chern-Simons term $\left(k_{A F}\right)^{\alpha} A^{\beta} \tilde{F}_{\alpha \beta}$ preserves translation invariance, since $\left(k_{A F}\right)^{\mu}$ is assumed to be constant in the minimal SME. However, $\left(k_{A F}\right)^{\mu}$ has mass dimensions suggesting that dilatation and conformal symmetry are violated. One further expects 
the Lorentz group to be broken down to the appropriate three-dimensional little group associated with $\left(k_{A F}\right)^{\mu}$. This suggests that the MCS model maintains seven spacetime symmetries - four translations and three (of the original six) Lorentz invariances. This section argues that there is, in fact, one additional spacetime symmetry if $\left(k_{A F}\right)^{\mu}$ is lightlike: a combination of a boost and a dilatation.

We begin by streamlining our notation $\left(k_{A F}\right)^{\mu} \rightarrow k^{\mu}=(k, \vec{k})$ and by recalling that a dilatation, also called a scale transformation, takes $A^{\mu}(x) \rightarrow$ $e^{\rho} A^{\mu}\left(e^{\rho} x\right)$, where the size of the dilatation is determined by the parameter $\rho$. A dilatation therefore takes

$$
\int d^{4} x \mathcal{L}_{\mathrm{MCS}} \rightarrow \int d^{4} x\left(\mathcal{L}_{\mathrm{M}}+e^{-\rho} \mathcal{L}_{\mathrm{CS}}\right) \neq \int d^{4} x \mathcal{L}_{\mathrm{MCS}},
$$

where a suitable change of integration variables is understood. It is apparent that the conventional piece $\mathcal{L}_{\mathrm{M}}$ and the Chern-Simons extension $\mathcal{L}_{\mathrm{CS}}$ transform differently. Moreover, the difference between the original and the transformed actions is not a boundary term establishing the non-invariance of $\mathcal{L}_{\text {MCS }}$ under dilatations.

We next look at Lorentz transformations, which can be implemented via $\Lambda_{\nu}^{\mu}(\vec{\theta}, \vec{\beta})$. Here, $\vec{\theta}$ and $\vec{\beta}$ characterize rotations and boosts, respectively. Under such transformations, the MCS Lagrangian changes according to $\mathcal{L}_{\mathrm{MCS}}=\mathcal{L}_{\mathrm{M}}+\mathcal{L}_{\mathrm{CS}} \rightarrow \mathcal{L}_{\mathrm{M}}+\Lambda^{\mu}{ }_{\gamma}(-\vec{\theta},-\vec{\beta}) k^{\gamma} A^{\nu} \tilde{F}_{\mu \nu}$. We have again suppressed the the dummy integration variables $x$. Next, we consider a special boost: $\vec{\beta}=\beta \hat{k}$, where $\hat{k} \equiv \vec{k} /|\vec{k}|$. Such a boost scales $k^{\mu}$ by a factor of $e^{\beta}$, so that $\Lambda_{\gamma}^{\mu}(\overrightarrow{0},-\beta \hat{k}) k^{\gamma} A^{\nu} \tilde{F}_{\mu \nu}=\exp (\beta) k^{\mu} A^{\nu} \tilde{F}_{\mu \nu} \neq \mathcal{L}_{\mathrm{CS}}$ yielding

$$
\mathcal{L}_{\mathrm{MCS}} \rightarrow \mathcal{L}_{\mathrm{M}}+e^{\beta} \mathcal{L}_{\mathrm{CS}} \neq \mathcal{L} .
$$

This shows that symmetry under boosts along $\hat{k}$ is broken, as expected.

Although each individual transformation (4) and (5) no longer determines a symmetry, the specific form of these transformations shows that a dilatation combined with a suitable boost along the spatial direction of a lightlike $k^{\mu}$ remains an invariance of $\mathcal{L}_{\text {MCS }}$. We can verify this explicitly by studying the currents

$$
D^{\mu} \equiv \theta^{\mu \nu} x_{\nu}
$$

and

$$
J_{\alpha \beta}^{\mu} \equiv \theta_{\alpha}^{\mu} x_{\beta}-\theta_{\beta}^{\mu} x_{\alpha} .
$$

These are the usual dilatation and Lorentz currents, respectivly. To extract from Eq. (7) the current corresponding to a boost along $\hat{k}$, we decompose 
$k^{\mu}$ into its purely timelike and its purely spacelike part $k^{\mu}=k\left(k_{T}^{\mu}+k_{S}^{\mu}\right)$, where $k_{T}^{\mu}=(1, \overrightarrow{0})$ and $k_{S}^{\mu}=(0, \hat{k})$. The desired current component is then given by $J_{\alpha \beta}^{\mu} k_{S}^{\alpha} k_{T}^{\beta}$. The divergences of these currents satisfy

$$
\partial_{\mu} D^{\mu}=-\mathcal{L}_{\mathrm{CS}}
$$

and

$$
\partial_{\mu} J_{\alpha \beta}^{\mu} k_{S}^{\alpha} k_{T}^{\beta}=+\mathcal{L}_{\mathrm{CS}} .
$$

It again becomes clear that $D^{\mu}$ and $J_{\alpha \beta}^{\mu} k_{S}^{\alpha} k_{T}^{\beta}$ are not conserved individually. However, their sum $Q^{\mu} \equiv D^{\mu}+J_{\alpha \beta}^{\mu} k_{S}^{\alpha} k_{T}^{\beta}$ is, in fact, conserved. An explicit gauge-invariant expression for $Q^{\mu}$ can be obtained: ${ }^{12}$

$$
Q^{\mu}=\left[\frac{1}{4} \eta_{\nu}^{\mu} F^{2}+F^{\mu \alpha} F_{\alpha \nu}\right]\left[x^{\nu}+\left(k_{T} \cdot x\right) k_{S}^{\nu}-\left(k_{S} \cdot x\right) k_{T}^{\nu}\right] .
$$

We can thus see that in the lightlike MCS model an additional conserved current relative to the spacelike and timelike cases exists. With Killingvector techniques, one can show that this is the only additional conservation law in the present context. ${ }^{12}$ This extended symmetry structure is described by the Lie algebra $\operatorname{sim}(2){ }^{12}$

\section{Vacuum Cherenkov radiation in the MCS model}

The Lorentz- and CPT-violating SME coefficients act in many respects like a background. This analogy is particularly well suited for the electrodynamics sector of the SME because this sector exhibits many parallels to the conventional Maxwell theory in macroscopic media. It is therefore natural to ask as to whether such analogies can be exploited to identify possible phenomenological Lorentz- and CPT-breaking effects in the SME that can be employed for tests.

One conventional effect inside a macroscopic medium is that the phase speed of light $c_{p h}$ can be slowed down relative to the vacuum $c>c_{p h}$. It then becomes possible for ordinary charges $q$ to travel faster than light inside this medium:

$$
v_{q}>c_{p h} \equiv \frac{\omega}{|\vec{p}|}
$$

Here, $v_{q}$ is the charge's speed, $\omega$ the photon frequency, and $\vec{p}$ the photon wave vector. It turns out this configuration is unstable in the sense that these fast charges are decelerated rapidly through the emission of photons. This well established effect is called Cherenkov radiation. It can be employed, for example, in modern particle detectors. 
Can the Cherenkov effect also occur in our MCS model? This is indeed the case, which can be seen as follows. One can verify ${ }^{15}$ that Condition (11) continues to hold in the presence of Lorentz and CPT violation. It follows that the MCS plane-wave dispersion relation $\omega=\omega(\vec{p})$ must be investigated. This dispersion relation is determined by

$$
p^{4}+4 k^{2} p^{2}-4(k \cdot p)^{2}=0,
$$

where $p^{\mu}=(\omega, \vec{p})$. For a given $\vec{p}$, Eq. (12) determines a quartic equation in $\omega$, so that there are four branches of solutions. Two of these branches lie inside the momentum-space lightcone where $\omega(\vec{p})>\vec{p}$, which is inconsistent with Requirement (11). However, the other two branches are located outside the lightcone where $\omega(\vec{p})<\vec{p}$ and the Cherenkov condition (11) is satisfied. We conclude that the Cherenkov effect can indeed occur in vacuum within the context of the MCS model.

An important criterion for experimental Lorentz tests is the rate for vacuum Cherenkov radiation. Within the MCS model, a classical calculation treating the charge as an external source yields ${ }^{15}$

$$
\dot{P}^{\mu}=-\operatorname{sgn}\left(k_{0}\right) \frac{q^{2}}{4 \pi} \frac{k_{0}^{4}}{\vec{k}^{2}}(0, \hat{k})
$$

in the charge's rest frame. Here, $\dot{P}^{\mu}$ is the rate of four-momentum radiation and $q$ the particle's charge. It is apparent that the rate is suppressed: it is second order in the SME coefficient $k^{\mu}$. In the MCS model, vacuum Cherenkov radiation is therefore phenomenologically less interesting.

\section{References}

1. See, e.g., V.A. Kostelecký and S. Samuel, Phys. Rev. D 39, 683 (1989); V.A. Kostelecký and R. Potting, Nucl. Phys. B 359, 545 (1991); B. Altschul and V.A. Kostelecký, Phys. Lett. B 628, 106 (2005).

2. See, e.g., S.M. Carroll et al., Phys. Rev. Lett. 87, 141601 (2001); Z. Guralnik et al., Phys. Lett. B 517, 450 (2001); C.E. Carlson et al., Phys. Lett. B 518, 201 (2001); A. Anisimov et al., Phys. Rev. D 65, 085032 (2002).

3. V.A. Kostelecký et al., Phys. Rev. D 68, 123511 (2003); R. Jackiw and S.-Y. Pi, Phys. Rev. D 68, 104012 (2003); O. Bertolami et al., Phys. Rev. D 69, 083513 (2004); N. Arkani-Hamed et al., JHEP 0507, 029 (2005).

4. J. Alfaro et al., Phys. Rev. D 66, 124006 (2002); D. Sudarsky et al., Phys. Rev. Lett. 89, 231301 (2002); G. Amelino-Camelia, Mod. Phys. Lett. A 17, 899 (2002); R.C. Myers and M. Pospelov, Phys. Rev. Lett. 90, 211601 (2003); N.E. Mavromatos, Lect. Notes Phys. 669, 245 (2005).

5. F.R. Klinkhamer, Nucl. Phys. B 578, 277 (2000); F.R. Klinkhamer and J. Schimmel, Nucl. Phys. B 639, 241 (2002). 
6. C.D. Froggatt and H.B. Nielsen, arXiv:hep-ph/0211106.

7. J.D. Bjorken, Phys. Rev. D 67, 043508 (2003).

8. C.P. Burgess et al., JHEP 0203, 043 (2002); A.R. Frey, JHEP 0304, 012 (2003); J. Cline and L. Valcárcel, JHEP 0403, 032 (2004).

9. D. Colladay and V.A. Kostelecký, Phys. Rev. D 55, 6760 (1997); Phys. Rev. D 58, 116002 (1998); V.A. Kostelecký and R. Lehnert, Phys. Rev. D 63, 065008 (2001).

10. V.A. Kostelecký, Phys. Rev. D 69, 105009 (2004); R. Bluhm and V.A. Kostelecký, Phys. Rev. D 71, 065008 (2005).

11. See, e.g., R. Jackiw and V.A. Kostelecký, Phys. Rev. Lett. 82, 3572 (1999); V.A. Kostelecký et al., Phys. Rev. D 65, 056006 (2002); R. Lehnert, Phys. Rev. D 68, 085003 (2003); J. Math. Phys. 45, 3399 (2004); Phys. Rev. D 74, 125001 (2006); V.A. Kostelecký and R. Potting, Gen. Rel. Grav. 37, 1675 (2005); B. Altschul, Phys. Rev. D 73, 045004 (2006); J. Phys. A 39, 13757 (2006).

12. A.J. Hariton and R. Lehnert, Phys. Lett. A 367, 11 (2007).

13. See, e.g., this proceedings; V.A. Kostelecký, ed., CPT and Lorentz Symmetry III, World Scientific, Singapore, 2005; D. Mattingly, Living Rev. Rel. 8, 5 (2005).

14. See, e.g., J. Lipa et al., Phys. Rev. Lett. 90, 060403 (2003); P.L. Stanwix et al., Phys. Rev. D 74, 081101 (2006); M.E. Tobar et al., Phys. Rev. D 71, 025004 (2005); M. Hohensee et al., Phys. Rev. D 75, 049902 (2007); J.P. Cotter and B. Varcoe, physics/0603111; H. Müller et al., arXiv:0706.2031.

15. See, e.g., R. Lehnert and R. Potting, Phys. Rev. Lett. 93, 110402 (2004); Phys. Rev. D 70, 125010 (2004); C. Kaufhold and F.R. Klinkhamer, Nucl. Phys. B 734, 1 (2006); B. Altschul, Phys. Rev. Lett. 98, 041603 (2007).

16. See, e.g., T. Katori and R. Tayloe, in Ref. 13; LSND Collaboration, L.B. Auerbach et al., Phys. Rev. D 72, 076004 (2005); V.A. Kostelecký and M. Mewes, Phys. Rev. D 69, 016005 (2004); V. Barger et al., arXiv:0706.1085 [hep-ph].

17. See, e.g., H. Dehmelt et al., Phys. Rev. Lett. 83, 4694 (1999); G. Gabrielse et al., Phys. Rev. Lett. 82, 3198 (1999); L.-S. Hou, W.-T. Ni, and Y.-C.M. Li, Phys. Rev. Lett. 90, 201101 (2003); H. Müller, Phys. Rev. D 71, 045004 (2005).

18. See, e.g., D.F. Phillips et al., Phys. Rev. D 63, 111101(R) (2001); P. Wolf et al., Phys. Rev. Lett. 96, 060801 (2006); O. Bertolami et al., Phys. Lett. B 395, 178 (1997).

19. See, e.g., BELLE Collaboration, K. Abe et al., Phys. Rev. Lett. 86, 3228 (2001); BaBar Collaboration, B. Aubert et al., Phys. Rev. Lett. 92, 181801 (2004); arXiv:hep-ex/0607103; FOCUS Collaboration, J.M. Link et al., Phys. Lett. B 556, 7 (2003).

20. V.W. Hughes et al., Phys. Rev. Lett. 87, 111804 (2001); R. Bluhm et al., Phys. Rev. Lett. 84, 1098 (2000).

21. S.M. Carroll et al., Phys. Rev. D 41, 1231 (1990). 